\title{
Quantum Cryptography over Underground Optical Fibers
}

\author{
R. J. Hughes, G. G. Luther, G. L. Morgan, C. G. Peterson and C. Simmons \\ University of California, \\ Physics Division, \\ Los Alamos National Laboratory, \\ Los Alamos, NM 87545, USA
}

\begin{abstract}
Quantum cryptography is an emerging technology in which two parties may simultaneously generate shared, secret cryptographic key material using the transmission of quantum states of light whose security is based on the inviolability of the laws of quantum mechanics. An adversary can neither successfully tap the key transmissions, nor evade detection, owing to Heisenberg's uncertainty principle. In this paper we describe the theory of quantum cryplography, and the most recent results from our experimental system with which we are generating key material over $14-\mathrm{km}$ of underground optical fiber. These results demonstrate that optical-fiber based quantum cryptography could allow secure, real-time key generation over "open" multi-km node-to-node optical fiber communications links between secure "islands."
\end{abstract}

\section{Introduction}

Two of the main goals of cryptography (encryption and authentication of messages) can be accomplished, with provable security, if the sender ("Alice") and recipient ("Bob") possess a secret random bit sequence known as "key" material. The initial step of key distribution, in which the two parties acquire the key material, must be accomplished with a high level of confidence that a third party ("Eve") cannot acquire even partial information about the random bit sequence. If Alice and Bob communicate solely through classical messages it is impossible for them to generate a certifiably secret key owing to the possibility of passive eavesdropping. However, secure key distribution becomes possible if they communicate with single-photon transmissions using the emerging technology of quantum cryptography, or more accurately, quantum key distribution (QKD).[1] (A small amount of shared secret key material is required to initialize the system.)

The security of QKD is based on the inviolability of the laws of quantum mechanics. An adversary cannot "tap" the key transmissions owing to the indivisibility of quanta. At a deeper level, QKD resists interception and retransmission by an eavesdropper because in quantum mechanics, in contrast to the classical world, the result of a measurement cannot be thought of as revealing a "possessed value" of a quantum state. Furthermore, a unique aspect of quantum cryptography is that Heisenberg's uncertainty principle ensures that an eavesdropper's activities must produce 
an irreversible change in the quantum states ("collapse of the wavefunction") before they are retransmitted to the intended recipient. These changes will introduce an anomalously high error rate in the transmissions between the sender and intended recipient, allowing them to detect the altempted eavesdropping.

Because it has the ultimate security assurance of a law of Nature quantum cryptography offers potentially attractive "case of use" advantages over conventional key distribution schemes: it avoids the "insider threat" because key material does not exist before the quantum transmissions take place; it avoids the cumbersome physical security aspects of conventional key distribution methods; and it provides a secure alternative to key distribution schemes based on public key cryptography, which are becoming vulnerable to algorithmic advances and improved computing techniques.[2] Thus, quantum key distribution enables "encrypted communications on demand," because it allows key generation at transmission time over an unsecure optical communications link.

The origins of quantum cryptography can be traced to the work of Wiesner in the early 1970s, who proposed that if single-quantum states could be stored for long periods of time they could be used as counterfeit-proof money. Wiesner eventually published his ideas in 1983,[3] but they were of largely academic interest owing to the impracticality of isolating a quantum state from the environment for long time periods. However, Bennett and Brassard realized that instead of using single quanta for information storage they could be used for information transmission. In 1984 they published the first quantum cryptography protocol now known as "BB84".[4] A further advance in theoretical quantum cryptography took place in 1991 when Ekert proposed[5] that Einstein-Podolsky-Rosen (EPR) "entangled" twoparticle states could be used to implement a quantum cryptography protocol whose security was based on Bell's inequalities. Starting in 1989, Bennett, Brassard and collaborators demonstrated that QKD was potentially practical by constructing a working prototype system for the BB84 protocol, using polarized photons.[6] Although the propagation distance was only about $30 \mathrm{~cm}$, this experiment is in several ways still the most thorough demonstration of quantum cryptography.

In 1992 Bennett published a "minimal" QKD scheme ("B92") and proposed that it could be implemented using single-photon interference with photons propagating for long distances over optical fibers.[7] Since then, experimental groups in the UK, [8] Switzerland[9] and the USA[10,11] have developed optical fiber-based prototype QKD systems. The aim of these experiments has been to show the conceptual feasibility of QKD, rather than to produce the definitive system, or to address a particular cryptographic application. However, we have demonstrated the feasibility of low-error rate QKD over underground optical fibers that were installed for network applications. We have been generating key material over $14 \mathrm{~km}$ of fiber throughout 1995 and we anticipate increasing the propagation distance to $24 \mathrm{~km}$ and possibly to $31 \mathrm{~km}$ during early 1996.

The remainder of this paper is organized as follows. In section 2 we give a concise introduction to the theory of quantum cryptography. Then, in section 3 we describe the experimental considerations underlying our implementation of quantum cryptography in optical fibers and the performance of our system. Finally, in section 4 we present some conclusions. 


\section{Quantum cryptography: theory}

To understand QKD we must first move away from the traditional key distribution metaphor of Alice sending particular key data to Bob. Instead, we should have in mind a more symmetrical starting point, in which Alice and Bob initially generate their own, independent random number sets, containing more numbers than they need for the key material that they will ultimately share. Next, they compare these sets of numbers to distill a shared subset, which will become the key material. It is important to appreciate that they do not need to identify all of their shared numbers, or even particular ones, because the only requirements on the key material are that the numbers should be secret and random. They can attempt to accomplish a secret distillation if Alice prepares a sequence of tokens, one kind for a " 0 " and a different kind for a " 1 ", and sends a token to Bob for each bit in her set. Bob proceeds through his set bit-by-bit in synchronization with Alice, and compares Alice's token with his bit. He then replies to Alice telling her whether the token is the same as his number (but not the value of his bit). With Bob's information Alice and Bob can identify bits they have in common. They keep these bits, forming the key, and discard the others. If one of Alice's tokens fails to reach Bob this does not spoil the procedure, because it is only tokens that arrive which are used in the distillation process.

The obvious problem with this procedure is that if the tokens are classical objects they carry the bit values before they are observed by Bob, and so they could be passively monitored by Eve. However, it is possible to overcome this problem and generate a secure key by using "non-orthogonal" quantum states as the tokens. Several QKD protocols have been developed, but for simplicity we shall describe the minimal B92 QKD protocol[7] in terms of the preparation and measurement of single-photon polarization states.

We will first review some of the salient features of the quantum mechanics of polarized light. In classical physics light of a single color is described by an electromagnetic field in which electric and magnetic fields oscillate at a frequency, $v$, that is related to the wavelength, $\lambda$, by the relation $c=v \lambda$, where $c$ is the velocity of light. For example, visible light has wavelengths in the range from $400-750 \mathrm{~nm}$, while longer wavelength radiation (invisible to the eye) is known as infra-red. An important property of optical waves is their polarization: we will define a vertically polarized ("V") wave as one for which the electric field is restricted to lie along the $z$-axis for a wave propagating along the $x$-axis, and similarly a horizontally polarized (" $\mathrm{H}$ ") wave will be defined as one in which the electric field lies along the $y$-axis. It is a remarkable property of light that any other polarization state of light propagating along the $x$-axis can be resolved into a linear superposition of vertically polarized and horizontally polarized waves with a particular relative phase. In the case of linearly polarized light the amplitude of the two components is determined by the projections of the polarization direction along the $\mathrm{V}$ or $\mathrm{H}$ polarization axes. For instance, light linearly polarized along the $+45^{\circ}$ direction in the $y-z$ plane is an equal amplitude, in-phase superposition of $\mathrm{V}$ and $\mathrm{H}$, while light polarized along the $-45^{\circ}$ direction in the $y-z$ plane is an equal amplitude, opposite-phase superposition. For 
obvious reasons $\mathrm{V}$ and $\mathrm{H}$ polarization states (or $+45^{\circ}$ and $-45^{\circ}$ polarizations) will be referred to as orthogonal polarizations, while two polarizations (such as $V$ and $+45^{\circ}$ ) that have a non-zero projection will be called non-orthogonal.

Light of a particular linear polarization can be produced by sending unpolarized light through a polarizing medium (Polaroid) whose polarizing axis is oriented along the direction of the desired linear polarization. When this light is passed through a second polarizer, only the component polarized parallel to the polarizing axis emerges, while the orthogonal component is absorbed. For example if $\mathrm{V}$ light impinges on a polarizer oriented at $+45^{\circ}$ the emerging light is reduced in amplitude by a factor of $2^{-1 / 2}$, has the $+45^{\circ}$ polarization and an intensity (proportional to the square of the amplitude) which is $50 \%$ of the incident intensity. Likewise, if $\mathrm{V}$ light impinges on an $\mathrm{H}$ polarizer, no light emerges, and we refer to this configuration as having "crossed polarizers."

The foregoing summarizes the essential features of classical polarized light, but in quantum cryptography we deal with very low intensity light where quantum mechanics must be used. Specifically, during propagation such light has wave-like properties, but on detection exhibits particle-like behavior: the optical energy is quantized into indivisible units of size $h \mathrm{v}$, called photons (the elementary particles of electromagnetic radiation) where $h$ is a fundamental constant of Nature known as Planck's constant. The indivisibility of photons raises the interesting question of how a photon of polarized light behaves when it encounters a polarizer. Clearly, there is no difficulty of interpretation if we are only concerned with orthogonal polarizations: a V-photon would pass a V-polarizer with certainty, but be absorbed by an $\mathrm{H}$-polarizer with certainty, for instance. However, once non-orthogonal polarizations are introduced the peculiarities of quantum mechanics become evident. Consider a $+45^{\circ}$-polarized photon impinging on a $\mathrm{V}$-polarizer: there is no such object as a "half photon" by analogy with the classical 50\% transmission intensity. Instead, quantum mechanics predicts that there is a $50 \%$ probability that the photon will be absorbed, and a $50 \%$ probability that it will be transmitted, with V-polarization, in any given trial (experiment). Of particular relevance for quantum cryptography is that beyond these probabilities we cannot predict how a particular $+45^{\circ}$-photon will behave at a V-polarizer. (Of course the result of a given experiment is either a definite absorption or transmission, but with many repetitions of the experiment we would build up a set of results reflecting the 50-50 absorption-transmission probabilities.) A further relevant quantum peculiarity is that if a $+45^{\circ}$-photon passes the V-polarizer it loses all of its " $+45^{\circ}$-ness." Specifically, if the emerging photon is made to impinge on a second polarizer oriented at $+45^{\circ}$ it will be absorbed or transmitted with $50 \%$ probability in each case, even though it was originally a $+45^{\circ}$ photon. This randomization of properties by non-orthogonal measurements is a reflection of Heisenberg's uncertainty principle, and is a crucial element in the detectability of eavesdropping in quantum cryplography. In the terminology of quantum mechanics one says that the V-polarizer has "collapsed the photon's wavefunction."

In the B92 QKD protocol Alice can produce photons with either of two nonorthogonal polarizations: $\mathrm{V}$ or $+45^{\circ}$ and Bob can make either of two non-orthogonal 
polarization measurements: $-45^{\circ}$ or $\mathrm{H}$. The first step of the protocol is for Alice and Bob to generate their own independent sets of random binary numbers. In the second step they proceed through their sets bit-by-bit in synchronization, with Alice preparing a polarized photon for each of her bits according to the rules:

$$
\begin{gathered}
\text { "0"↔V } \\
" 1 " \leftrightarrow+45^{\circ}
\end{gathered} .
$$

Alice sends each photon over a "quantum channel" to Bob. (The quantum channel is a transmission medium that isolates the quantum state from interactions with the "environment.") Next, Bob makes a polarization measurement on each photon he receives, according to the value of his bit as given by:

$$
\begin{gathered}
" 0 " \leftrightarrow-45^{\circ} \\
" 1 " \leftrightarrow H
\end{gathered}
$$

and records the result ("pass" $=\mathrm{Y}$, "fail" $=\mathrm{N}$ ). Note that Bob will never record a "pass" if his bit is different from Alice's (crossed polarizers), and that he records a "pass" on $50 \%$ of the bits that they have in common. In the example in Figure 1

\begin{tabular}{|c|c|c|c|c|}
\hline Alice's numbers & 1 & 0 & 1 & 0 \\
\hline Alice's polarization & $+45^{\circ}$ & $\mathrm{V}$ & $+45^{\circ}$ & $\mathrm{V}$ \\
\hline Bob's polarization & $-45^{\circ}$ & $-45^{\circ}$ & $\mathrm{H}$ & $\mathrm{H}$ \\
\hline Bob's numbers & 0 & 0 & 1 & 1 \\
\hline Bob's results & $\mathrm{N}$ & $\mathrm{N}$ & $\mathrm{Y}$ & $\mathrm{N}$ \\
\hline
\end{tabular}

Figure 1. An example of 892 quantum key distribution

we see that for the first and fourth bits Alice and Bob had different bit values, so that Bob's result is a definite "fail" in each case. However, for the second and third bits, Alice and Bob have the same bit values and the protocol is such that there is a probability of 0.5 that Bob's result is a "pass" in each case. Of course, we cannot predict in any particular experiment which one will be a "pass," but in this example the second bit was a "fail" and the third bit was a "pass."

To complete the protocol Bob sends a copy of his results to Alice, but not the measurement that he made on each bit. (It is at this data reconciliation stage that the initial key material is required for authentication. This key material can be replaced by a portion of the kcy material generated by QKD.) He may send this information over a conventional (public) channel which may be subject to eavesdropping. Now Alice and Bob retain only those bits for which Bob's result was " $Y$ " and these bits become the shared key material. (In the example of Figure 1 the third bit becomes the first bit of the shared key.) This procedure distills one shared bit from four initial bits because it only identifies $50 \%$ of the bits that Alice and Bob actually have in common. However, this inefficiency is the price that Alice and Bob must pay for secrecy. 
An eavesdropper performing her own measurement of Alice's states on the quantum channel (using Bob's measurement basis for example) will introduce a $25 \%$ error rate between Alice and Bob's key material owing to the phenomenon of wavefunction collapse described earlier. Alice and Bob can test for eavesdropping by agreeing to sacrifice a portion of their key material to test for the error rate. If this error rate is as high as $25 \%$ they will know that Eve has been monitoring their transmissions and that they should discard the whole set of key material. In practice, if the error rate is acceptably low Alice and Bob can use this information to implement a suitable error correction procedure to remove errors arising from experimental imperfections. This can then be followed by a further stage of "privacy amplification" to reduce any partial knowledge acquired by Eve to an arbitrarily low level.[12]

Traditionally it has been proposed that the key bits generated by QKD should be used for the encryption of communications using the unbreakable "onetime pad" method. However, the key material could equally well (and more practically) be used by Alice and Bob in any other symmetric key cryptosystem. For example, they could use a short string of their key bits as an input "seed" to a cryptographically secure random number generator, whose output would provide a "key expansion" to many secure bits for use in subsequent encryption.

\section{Quantum cryptography: experimental realization}

Although single-photon polarization states are a convenient way to describe QKD any two-state quantum system may be used for QKD. Single-photon states which are more suited to long propagation distances in optical fibers can be constructed by allowing a photon to impinge on a beamsplitter. Alice and Bob may construct this interferometric version of QKD if Alice has a source of single photons that she can inject into a Mach-Zehnder interferometer in which she controls the phase, $\phi_{A}$, along one of the optical paths. (In this context the optical phase is an angular expression of the length of the optical path: a phase change of $2 \pi$ corresponds to a change in path length of one optical wavelength.) Bob has a single-photon detector at one of the output ports and controls the phase, $\phi_{B}$, along the other optical path.[1] (Sce Figure 2 in which we have indicated the sequence of optical phases corresponding to the bit sequences in the example of section 2.)

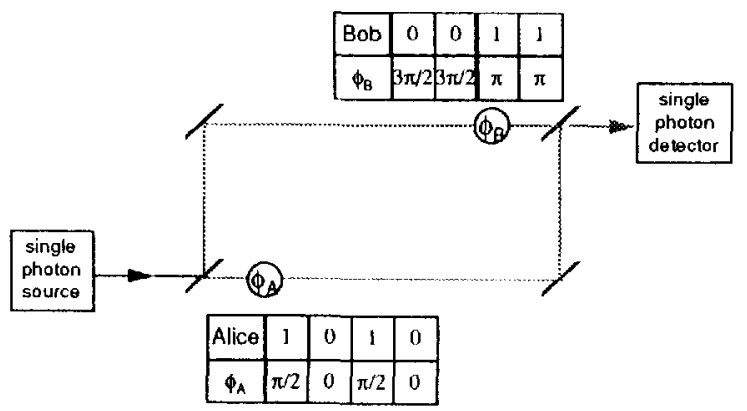

Figure 2. An interferometric realization of B92 quantum key distribution. 
Although a photon behaves as a particle on detection it propagates through the interferometer as a wave. The probability that a photon injected by Alice is detected by Bob

$$
P_{D}=\cos ^{2}\left(\frac{\phi_{A}-\phi_{B}}{2}\right),
$$

depends on both paths and exhibits the phenomenon of single-photon interference between the propagation amplitudes for the upper and lower paths: the detection probability varies between 1 (equal path lengths, constructive interference) and 0 (path difference of half a wavelength, destructive interference). Of course, total probability is conserved, and the remaining probability corresponds to the photon emerging from Bob's unused exit port of the interferometer. Thus, if Alice and Bob use the phase angles $\left(\phi_{A}, \phi_{B}\right)=(0,3 \pi / 2)$ for their " 0 " bits (respectively) and $\left(\phi_{A}, \phi_{B}\right)=(\pi / 2, \pi)$ for their "1" bits they have an exact representation of B92. Each path length is analogous to one of the polarizer angles in the explanation of $\mathrm{B} 92$ in the previous section. (Other single-particle QKD protocols, such as BB84,[4] can be realized with different choices for the phase angles.)

To construct a practical quantum cryptography device using single-photon interference we must consider the propagation medium and detection of "single photons." Optical fibers are an obvious choice because they are widely used in telecommunications and there are commercially available components, possibly allowing a system to be constructed that can perform quantum cryptography over an installed communications system. However, although optical fibers possess the good feature of guiding photons from source to detector, their properties largely determine the operating characteristics of a system. For example: what wavelength should we choose to operate at? Two factors are relevant to this question. At what wavelengths is single-photon detection possible with non-negligible efficiency? and at what wavelengths do optical fibers have low attenuation?

For photons in the wavelength range of $600-800 \mathrm{~nm}$ there are commercially available single-photon counting modules based on silicon ( $\mathrm{Si}$ ) avalanche photodiodes (APDs), which have high efficiencies and low noise rates. However, the attenuation of (single-mode) optical fibers is quite high in this wavelength range $(\sim 3$ $\mathrm{dB} / \mathrm{km}$ ), which will adversely affect the data rate and the noise rate if we choose to operate in this region. (The loss mechanism is predominantly Rayleigh scattering out of the fiber.) Conversely, optical fibers have much lower attenuation in the infra-red at the $1.3-\mu \mathrm{m}$ wavelength $(\sim 0.3 \mathrm{~dB} / \mathrm{km})$, and lower again at $1.55 \mu \mathrm{m}$, but although there are commercially available germanium (Ge) and indium-gallium arsenide (InGaAs) APDs that are sensitive to light at these infrared wavelengths, there are no commercially available single-photon counting modules. Nevertheless, several groups have shown that Ge APDs can detect single photons at $1.3 \mu \mathrm{m}$ if they are first cooled to reduce noise, and operated in so-called Geiger mode, in which they are biased above breakdown.[13] An incoming photon liberates an electron-hole pair, which with some probability initiates an avalanche current, whose detection signals the arrival of the photon. For our project we decided that the propagation distance 
advantages of the $1.3-\mu \mathrm{m}$ wavelength were such that we characterized the performance of several (Fujitsu) APDs (both Ge and InGaAs) for single-photon detection at this wavelength.

Several parameters are important in characterizing the detector performance: single-photon detection efficiency; intrinsic noise rate (dark counts); and time resolution. We measured absolute detection efficiencies of $10-40 \%$, (for InGaAs APDs), but noise rates that are $\sim 1,000$ times higher than for Si-APD photon counting modules at $800 \mathrm{~nm}$. (See Figure 3 for example.) However, our detectors also have very good time resolutions, which can be utilized to compensate for the higher intrinsic noise rate because of the low dispersion of optical fibers at $1.3 \mu \mathrm{m}$. Thus, if a $1.3-\mu \mathrm{m}$ photon is injected into a fiber in a short wavepacket (300-ps, say) it will emerge from the far end without being significantly delocalized and so, because we know that the photon will be expected within a short time window we need only consider the probability of a noise count in this short time interval. This probability is only $\sim 5 \times 10^{-6}$ at the $50-\mathrm{kHz}$ noise rate for $20 \%$ efficiency in the InGaAs device shown in Figure 3.

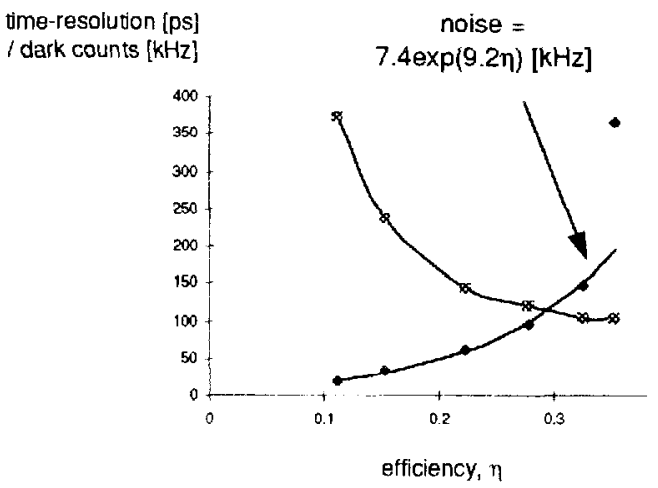

Figure 3. Geiger-mode operation of InGaAs avalanche photodiode: time-resolution and dark counts versus single-photon detection efficiency.

If we were to use different optical fibers for each of the interfering paths in the interferometric realization of $\mathrm{B} 92$ in Figure 2, we would have a very unstable interferometer for all but the shortest propagation distances. However, a more stable system can be produced by multiplexing both paths onto a single fiber in a design first proposed by Bennett.[7] In this design Alice and Bob have identical, unequalarm Mach-Zehnder interferometers with a "short" path and a "long" path, with one output port of Alice's interferometer optically coupled to one of the input ports of Bob's. The difference of the light travel times between the long and short paths, $\Delta T$, is much larger than the coherence time of the light source, so there can be no interference within each small interferometer. However, interference can occur within the coupled system (see Figure 4). 


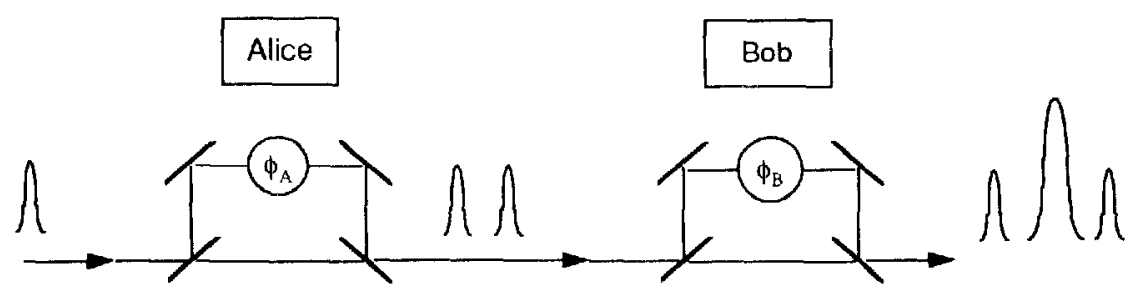

Figure 4. Time-multiplexed interferometer for quantum key distribution.

A photon injected into one of the input ports of Alice's interferometer from an attenuated pulsed laser source therefore has a $50 \%$ probability of entering Bob's interferometer, in a wave packet that is a coherent superposition of two pieces that are separated in time by $\Delta T$, corresponding to an amplitude for it to have taken the "short" path, and a delayed component which took the "long" path. On entering Bob's interferometer each component of the wave packet is again split into a "short" component and a "Iong" component, so that at each output port there are three "time windows" in which the photon may arrive. The first of these ("prompt") corresponds to the "short-short" propagation amplitude; which is followed after a delay of $\Delta T$ by the "central" component comprising the "short-long" and "long-short" amplitudes; and finally, after a further time $\Delta T$, the "delayed" time window corresponds to the "long-long" amplitude.

There is no interference in the "short-short" or "long-long" amplitudes, so the probability that the photon arrives in either of these time windows in either of Bob's output ports is $1 / 16$ (we assume $50 / 50$ beamsplitters and lossless mirrors). However, because the path-length differences in the two small interferometers are identical (to within the coherence length of the light source) interference does occur in the "central" time window between the "short-long" and "long-short" amplitudes. Indeed, because Alice and Bob can control the path length of their "long" paths with adjustable phases $\phi_{\mathrm{A}}$ or $\phi_{\mathrm{B}}$, respectively, the probability that the photon emerges in the "central" time window at the detector in the output port shown in Figure 4 is

$$
P=\frac{1}{8}\left[1+\cos \left(\phi_{A}-\phi_{B}\right)\right] \text {. }
$$

Note that within a factor of four this expression is identical with the photon arrival probability for the simple interferometric version of $\mathrm{B} 92$, and that, of the two interfering paths one ("long-short") is controlled by Alice and the other ("shortlong") is controlled by Bob just as in the simple interferometer of Figure 2. Thus, by sacrificing a factor of four in data rate this time-multiplexed interferometer can be used to implement QKD based on single-photon interference. (The photons "lost" in the prompt and delayed time windows are useful to test for a highly invasive Eve.)

We have constructed an optical fiber version of this time-multiplexed interferometer in which each of Alice's and Bob's interferometers are built from two 50/50 fiber couplers instead of beamsplitters. Each coupler has two input legs and two output legs: a photon entering on one leg has a $50 \%$ probability to cmerge from 
either of the output legs. No mirrors are required because the output fiber legs from the first coupler convey the photons to the input legs of the second coupler via a long fiber path or a short path $(\Delta T \sim 5 \mathrm{~ns})$. One of the output legs of Alice's interferometer is connected by a $14-\mathrm{km}$ long optical fiber path to one of the input legs of Bob's interferometer. (See Figure 5.) This $14-\mathrm{km}$ path is over underground optical fibers. Photons emerge from Alice's interferometer, located in our laboratory, and are conveyed through fiber jumpers to one of the underground fibers and thence to a remote location. At this far point the photons pass through more jumpers and back onto a second fiber for the return journey back to Bob's interferometer, which for convenience is also located in our laboratory. The total travel time over the underground link is about $67 \mu \mathrm{s}$, with $12.2 \mathrm{~dB}$ of attenuation owing to the 16 fiber connections along the path. This path represents a realistic Iest environment for quantum cryptography because of the diurnal temperature variations and other influences that could affect the photons' propagation that are outside of our control. Finally, photons emerge from one of the output legs of Bob's interferometer into a fiber pigtailed, cooled InGaAs APD detector.

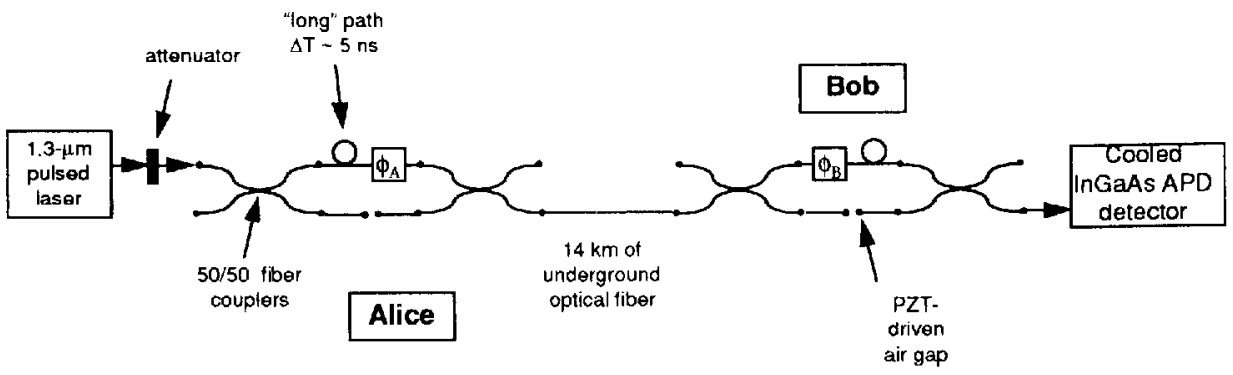

Figure 5. Schematic representation of the $14-\mathrm{km}$ quantum cryptography experiment.

A "single-photon" is generated by applying a 300 -ps electrical pulse with a $10-\mathrm{kHz}$ repetition rate to a low-power, fiber-pigtailed semiconductor laser whose output in then attenuated before coupling into the interferometer. Each "single-photon" pulse is preceded by a bright reference pulse, introduced from an optical impulse generator attached to the lower input leg of Alice's interferometer (not shown in Figure 5), to provide arrival time information to Bob. This bright pulse triggers a room-temperature detector in the upper output leg of Bob's interferometer (not shown), which provides the "start" signal for a time-interval analyzer and triggers the pulsed-bias gate signal to the cooled single-photon detector after a delay corresponding to the single-photon emission time relative to the bright pulse emission time. Single-photon arrival is indicated by the cold detector avalanche signal which also acts as the "stop" signal for the time interval analyzer. (Although Alice and Bob are located side-by-side in our laboratory there is no direct electrical connection between the sending and receiving electronics: their only links are the 14-km optical fiber "quantum channel" and the Ethernet "public channel" connection between their two independent computer control systems.) Figure 6 shows time-spectra of photon arrival times for four different phase differences that were set by driving airgaps located in the "short" paths with piezo-electric transducers (PZTs). 

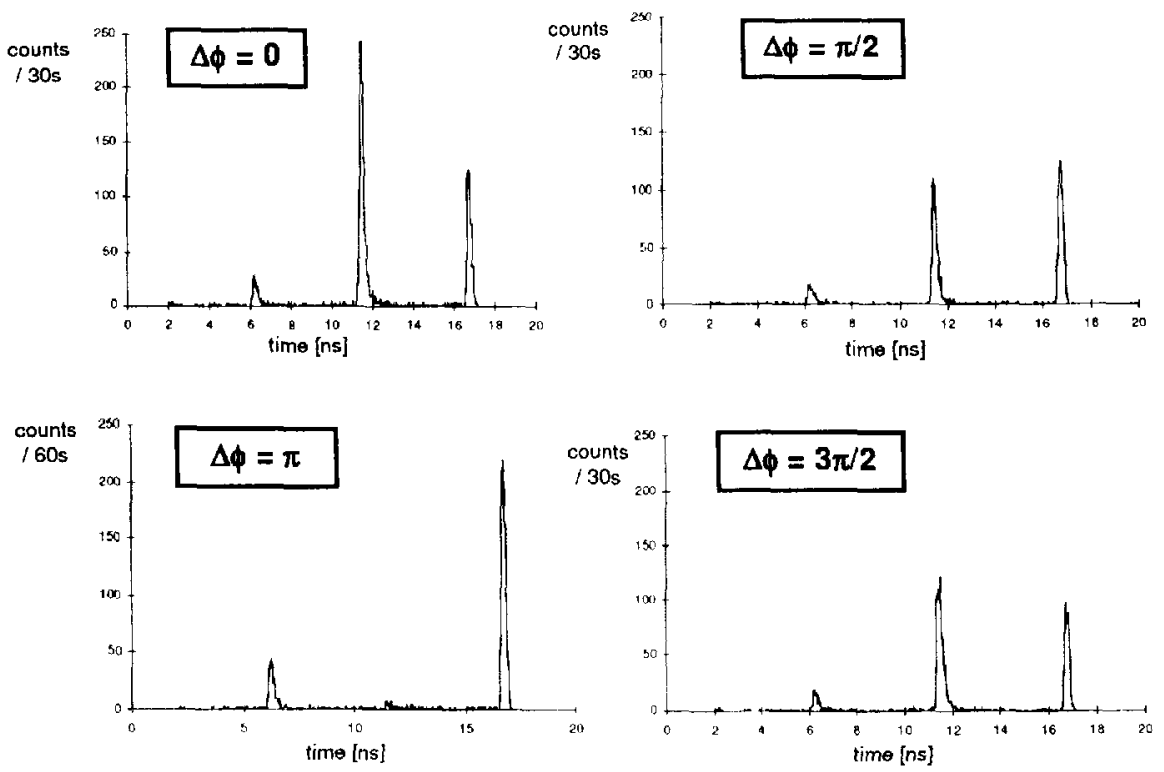

Figure 6. Photon time-of-arrival spectra accumulated at four phase difference values in the interferometer of Figure 5.

Photon counts were accumulated for $30 \mathrm{~s}$ at each phase setting, with the exception of the $\pi$-phase shift case, in which a 60 -s integration time was used, so as to collect more counts in the central peak. The 5 -ns scparation of the different paths is clearly visible, as is the 300-ps width of the laser pulse. The unequal height of the "short-short" (leftmost in each plot) and "long-long" (rightmost) peaks is due to attenuation in the air gaps. (This asymmetry is useful for detection of a "man-in-themiddle" attack by Eve.[7]) Polarization control was necessary within the interferometers in order to achieve the high visibility single-photon interference that is apparent in the central peak. The average number of photons per laser pulse arriving in the central peak maximum was $\bar{n}=0.04$, providing protection against a beamsplitting attack by Eve even if she was Iocated immediately after Alice's interferometer. Some noise counts are visible in the spectra, and after accumulating time spectra at other phase settings a background subtraction was performed on the central peaks, yielding an underlying interferometric visibility of $99.6 \pm 2.8 \%$. (i.e. when the path lengths differ by half a wavelength the interference is almost completely destructive.)

From the perspective of QKD this separation of the visibility is not as useful as the total probability of a count in the central time-window when the phase difference is $\pi$, because this quantity determines the error rate of the B92 protocol. (The probability that Bob detects a photon even though his bit value is different from Alice's determines the error rate.) Thus, the central peak in the second plot of Figure 6 represents 1264 received " $1-1$ "s generated from $3 \times 10^{5}$ initial " $1-1$ "s (a sequence of photons for which Alice and Bob both have the " 1 " bit value). The central peak of 
the fourth plot represents 1350 received " $0-0$ "s generated from $3 \times 10^{5}$ initial " $0-0$ "s; and the central peak of the third plot represents only 32 " $0-1$ " errors generated from $6 \times 10^{5}$ initial " 0 - 1 "s. These results give an inferred efficiency of key bit generation of $2.2 \times 10^{-3}$ per initial random bit, and a bit error rate (BER) of $1.2 \%$.

A B92 key generation procedure starts with two independent computer control systems (Alice and Bob) generating strings of pseudo-random numbers which are sequentially converted into voltages that are applied to either electro-optic phase modulators (for high speed key generation) or to the PZT-driven mirrors (for lowspeed key generation). (We use pseudo-random number generation for experimental convenience. A real QKD system would require true random number generation, which could be accomplished by utilizing detector noise for instance.) Detected photons are recorded by Bob's computer to identify bits shared with Alice. A file containing the detected photon bit positions is then communicated to Alice over an ethernet connection, enabling her to complete the key generation procedure. (BB84 key generation could also be implemented with a straightforward change of the computer control software.) A sample of key material is shown is Figure 7.

\begin{tabular}{|c|c|c|c|c|c|}
\hline$A$ & 01010011 & 01001000 & 01000100 & 10100010 & 10000101 \\
\hline B & 01010001 & 01001000 & 00000100 & 10100010 & 10000101 \\
\hline$A$ & 01101011 & 10101000 & 11100101 & 00000111 & 01110100 \\
\hline $\mathbf{B}$ & 01101011 & 10101000 & 11100101 & 00000011 & 01110100 \\
\hline A & 10011101 & 00011110 & 01011001 & 10000101 & 01110000 \\
\hline B & 10011101 & 00011110 & 01011001 & 10000101 & 01110000 \\
\hline A & 01111000 & 11101010 & 11100000 & 00010101 & 11100011 \\
\hline B & 01111000 & 11101010 & 11100000 & 00010101 & 11100011 \\
\hline A & 10011111 & 01100000 & 11000000 & 00100011 & 10000110 \\
\hline B & 10011101 & 01100000 & 11000000 & 00100011 & 10010110 \\
\hline $\mathbf{A}$ & 00010101 & 00000100 & 10010100 & 10000010 & 10100110 \\
\hline B & $0 \begin{array}{lllllllllllll}0 & 0 & 1 & 0 & 1 & 0 & 1\end{array}$ & 00000000 & 10010500 & 10000010 & 10100110 \\
\hline A & 00000010 & 01100001 & 011101100 & 11000010 & 11100001 \\
\hline B & 00000010 & 01100001 & 01100100 & 11000010 & 11100001 \\
\hline $\mathbf{A}$ & 11001110 & 01110010 & 10010100 & 01001010 & 01000100 \\
\hline H & 11001110 & 01110010 & 10010100 & 01001010 & 11000100 \\
\hline A & 10001110 & 11111111 & 01001011 & 000100011 & 11000110 \\
\hline B & 10001110 & 11111111 & 01001011 & 00010011 & 11000110 \\
\hline A & 10101100 & 10110101 & 10110000 & 01111000 & 01001101 \\
\hline B & 10101100 & 10110101 & 10110000 & 01111000 & 01001101 \\
\hline
\end{tabular}

Figure 7. A 400-bit sample of Alice's (A) and Bob's (B) key material generated by QKD

The key material above contains errors arising from visibility imperfections and detector noise. At present we remove these crrors by a simple block-parity check procedure. At this time we have not implemented a privacy amplification stage, but we do have a "one-time pad" encryption scheme in which we can encrypt short messages that are transmitted between the Alice and Bob computer systems over their Ethernet connection.

Several factors make the key generation rate of our QKD system considerably slower than the laser pulse rate. Firstly, the "single-photon" requirement intro- 
duces a reduction in rate because the majority of the laser pulses contain no photon. Then there are attenuation losses during propagation, which amount to about a factor of fifteen in our experiment. The QKD procedure itself has an intrinsic inefficiency of only identifying one shared bit from four initial bits, which is reduced by a further factor of four in our scheme, resulting in an additional factor of sixteen reduction in key rate. Finally, there is the detector efficiency to be included, which in our case was $20 \%$. There is a trade-off between key-rate, which increases with detector efficiency, and BER which also increases (exponentially) with efficiency. Thus for any specific distance there will be an optimal detection efficiency giving the least BER. In our experiment this would occur for $11 \%$ detection efficiency giving a BER of $1.1 \%$.

\section{Summary}

We have demonstrated that low error rate quantum cryptography is feasible over long distances $(14 \mathrm{~km})$ of installed optical fiber in a real-world environment, subject to uncontrolled temperature and mechanical influences. This represents an important step towards the practical feasibility of quantum cryptography. However, a complete, self-contained quantum cryptography system would be able to continuously generate secret key material in unattended mode over existing optical fibers. QKD could then be incorporated into existing information security systems so that users would be able to request an appropriate level of security for their needs and the system would deliver the corresponding quantity of key material for the particular encryption system that would be used. We are now working on the hardware and software developments requirements to achieve this goal.

\section{References}

1. For reviews see R. J. Hughes et al., "Quantum Cryptography," Contemporary Physics 36, 149 (1995); C. H. Bennett et al., "Quantum Cryptography," Scientific American 257 no.10, 50 (1992).

2. D. Atkins et al., "The Magic Words are Squeamish Ossifrage," Advances in Cryptology-ASIACRYPT '94 Proceedings, Springer-Verlag, 1995, pp.263.

3. S. Wiesner, "Conjugate Coding," SIGACT News 15, 78 (1983).

4. C. H. Bennett and G. Brassard, "Quantum Cryptography: Public Key Distribution and Coin Tossing," Proceedings of IEEE International Conference on Computers, Systems and Signal Processing, Bangalore (New York, IEEE, 1984).

5. A. K. Ekert, "Quantum Cryptography Based on Bell's Theorem," Phys. Rev. Lett. 67, 661 (1991).

6. C. H. Bennett and G. Brassard, "The Dawn of a New Era for Quantum Cryptography: The Experimental Prototype is Working," SIGACT NEWS 20, no. 4, 78 (1989); C. H. Bennett et al., "Experimental Quantum Cryptography," J. Crypto. 5, 3 (1992).

7. C. H. Bennett, "Quantum Cryptography Using Any Two Non-Orthogonal States," Phys. Rev. Lett. 68, 3121 (1992). 
8. P. D. Townsend, J. G. Rarity and P. Tapster, "Single Photon Interference in 10 km Long Optical Fiber Interferometer," Elec. Lett. 29, 634 (1994); P. D. Townsend, J. G. Rarity and P. Tapster, "Enhanced Single Photon Fringe Visibility in a 10-km Long Prototype Quantum Cryptography Channel," Elec. Lett. 29, 1291 (1994); P. D. Townsend, "Secure Key Distribution Based on Quantum Cryptography," Elec. Lett. 30, 809 (1994); C. Marand and P. D. Townsend, "Quantum Key Distribution Over Distances as Long as 30 km," Opt Lett. 20, 1695 (1995).

9. A. Muller et al., "Experimental Demonstration of Quantum Cryptography Using Polarized Photons in Optical Fibre Over More Than 1 km," Europhys. Lett. 23, 383 (1993); A. Muller, H. Zbinden and N. Gisin, "Underwater Quantum Coding," Nature 378, 449 (1995); A. Muller et al., "Quantum Cryptography Over $23 \mathrm{~km}$ in Installed Under-lake Telecom Fibre," Europhys. Lett. 33, 335 (1996).

10. J. D. Franson and H. Ilves, "Quantum Cryptography Using Optical Fibers," Appl. Optics 33, 2949 (1994).

11. R. J. Hughes et al., "Quantum Cryptography Over $14 \mathrm{~km}$ of Installed Optical Fiber," Los Alamos report LA-UR-95-2836, invited paper to appear in Proceedings of "Seventh Rochester Conference on Coherence and Quantum Optics," Rochester, NY, June 1995.

12. C. H. Bennett et al., "Generalized Privacy Amplification," IEEE Trans. Inf. Theory 41, 1915 (1995).

13. P. C. M. Owens et al., "Photon Counting Using Passively Quenched Germanium Avalanche Photodiodes," Appl. Optics 33, 6895 (1994); A. Lacaita et al., "Single-Photon Detection Beyond $1 \mu \mathrm{m}$ : Performance of Commercially Available Germanium Photodiodes," Appl. Optics 33, 6902 (1994). 